\title{
Asociación entre la Termografía por Infrarrojo, Parámetros Antropométricos y Gasto Energético de Reposo en Hombres
}

\author{
Association Between Infrared Thermography, Anthropometry \\ Parameters and Rest Energy Expenditure in Men
}

\author{
Barraza-Gómez Fernando, ${ }^{1,2}$; Alvear-Ordenes Ildefonso ${ }^{1,3}$; Hecht-Chau Gernot ${ }^{4}$; Henríquez Matías ${ }^{5}$ \& Tuesta Marcelo $^{6,7}$
}

BARRAZA-GÓMEZ, F.; ALVEAR-ORDENES, I.; HECHT-CHAU, G.; HENRÍQUEZ, M. \& TUESTA, M. Asociación entre la termografía por infrarrojo, parámetros antropométricos y gasto energético de reposo en hombres. Int. J. Morphol., 39(4):1176-1182, 2021.

RESUMEN: Es reconocido que un incremento de la grasa corporal subcutánea es capaz de alterar el patrón de liberación de calor a través de la piel. Sin embargo, la asociación con otras variables antropométricas y la influencia del gasto energético en reposo (GER), han sido escasamente abordadas. Este estudio determinó la relación de la temperatura de la piel con variables antropométricas, peso corporal y GER de hombres adultos sanos. Participaron en el estudio un total de 24 varones sanos de 33,9 $\pm 8,7$ años de edad, 85,8 $\pm 9,7 \mathrm{~kg}$ de peso corporal, $172,5 \pm 6,1 \mathrm{~cm}$ de estatura y $28,9 \pm 3,4 \mathrm{~kg} / \mathrm{m}^{2}$ de índice de masa corporal (IMC). Se realizaron evaluaciones antropométricas, de termografía por infrarrojo y del GER por calorimetría indirecta, con analizador de gases. La temperatura promedio de los 6-pliegues, y las individuales (toracoabdominales, supra-espinal y abdominal), tuvieron correlaciones negativas con el IMC, perímetro de cintura, índice cintura cadera, índice cintura estatura, y la sumatoria de 6 pliegues; todos con $p<0,05$. Además, la temperatura del pliegue supraespinal tuvo una correlación negativa con el porcentaje de masa adiposa (MA\%), $\mathrm{r}=0,47(\mathrm{p}=0,0194)$. La MA\% y el IMC tuvieron correlaciones negativas con el GER, con $\mathrm{r}=-0,59(\mathrm{p}=0,002)$ y $\mathrm{r}=-0,53(\mathrm{p}=0,006)$, respectivamente. El promedio de temperatura de los 6-pliegues presentó una correlación positiva con el GER $(r=0,44 ; p=0,02)$. La composición corporal causa una modificación en los patrones de termografía superficial local, sin afectar la relación entre el promedio de la temperatura superficial total con el GER, pudiendo éste ser un factor predictor.

PALABRAS CLAVE: Termometría; Espesor de pliegue cutáneo; Metabolismo; Índice de masa corporal.

\section{INTRODUCCIÓN}

Para mantener el equilibrio de la temperatura corporal, buena parte del calor producido por el metabolismo es eliminado desde la piel por radiación (Fernández-Cuevas et al., 2015; Salamunes et al., 2017). La termografía por infrarrojos (TI) es una tecnología que permite, entre otras, determinar la temperatura corporal superficial a través de la medición de la radiación corporal. La TI tiene la capacidad de detectar modificaciones y/o alteraciones, graves o no, de las funciones fisiológicas, permitiendo el estudio de quemaduras (Fernández-Cuevas et al.), cáncer (Herman \& Cetingul, 2011), daño muscular inducido por ejercicio (Gutiérrez-Vargas et al., 2017) o celulitis (Bauer et al., 2020). Además, en sujetos sanos y utilizando condi- ciones ambientales estables, la TI permite determinar el gasto energético en ejercicio (Jensen et al., 2016), estimar la fatiga (Hadzic et al., 2019) y observar los efectos de la modificación en la composición corporal (Law et al., 2018). La utilidad clínica de la TI se ha incrementado notablemente, por muchas razones; no es invasiva, ni compleja, es de bajo costo, bio-sostenible y de aplicación en tiempo real, permitiendo una monitorización continua (Hidalgo Salvador et al., 2014).

En el hombre se ha observado que la obesidad aumenta el calor corporal central. Un cambio provocado principalmente por el mayor aislamiento del tejido adiposo sub-

\footnotetext{
${ }^{1}$ Laboratorio de Fisiología Aplicada (FISAP), Universidad de León, Ponferrada, España.

${ }^{2}$ Universidad Viña del Mar, Chile.

${ }^{3}$ Instituto de Biomedicina (IBIOMED), Universidad de León, León, España.

${ }^{4}$ Departamento de Educación Física, Deportes y Recreación. Universidad Técnica Federico Santa María, Valparaíso, Chile.

${ }^{5}$ Instituto Nacional de Rehabilitación "Pedro Aguirre Cerda", Santiago de Chile, Chile.

${ }^{6}$ Escuela de Kinesiología, Universidad Andres Bello, Viña del Mar, Chile.

${ }^{7}$ Laboratorio de Ciencias del Deporte, Centro de Medicina Deportiva Sport MD, Viña del Mar, Chile.
} 
cutáneo (Salamunes et al.; Weigert et al., 2018), sobre todo en el esqueleto axial, con una desregulación vasoconstrictora periférica (Carrasco et al., 2002). Modificaciones provocadas por la distribución del tejido adiposo subcutáneo, que podrían modificar los patrones de eliminación de la temperatura corporal a través de la piel, variando la capacidad de disipación del calor producido por el metabolismo basal o durante el reposo. Por ejemplo, se ha demostrado que la obesidad disminuye la temperatura de la piel en el abdomen y muslo, mientras se observa aumentada en las palmas de las manos (Chudecka et al., 2014; Chudecka \& Lubkowska, 2015). Además, en hombres y mujeres, la temperatura promedio del pecho, espalda baja y espalda alta, descendía en la medida que los sujetos presentaban mayores porcentajes de grasa corporal total (Chudecka \& Lubkowska). Se ha observado también una relación entre la temperatura superficial en las zonas donde se han medido los pliegues cutáneos, siendo que a mayor grosor del pliegue subescapular, la temperatura superficial era menor en el pliegue y que también era mayor la diferencia entre la temperatura corporal central y la superficial de la misma zona (Neves et al., 2015). Hasta ahora, la disposición superficial del tejido adiposo y los niveles de temperatura han sido escasamente explorados y se desconocen los efectos que estos aspectos pueden tener sobre el gasto energético en reposo (GER). Por ello, el objetivo de este estudio fue determinar las relaciones entre la temperatura obtenida a partir de TI, variables antropométricas y el GER de hombres adultos sanos.

\section{MATERIAL Y MÉTODO}

Participantes. En este estudio participaron 24 hombres (edad: 33,9 \pm 8,7 años; masa corporal: 85,8 \pm 9,7 kg; estatura: $172,5 \pm 6,1 \mathrm{~cm})$, sanos, militares, no fumadores, pertenecientes a una base aérea de Chile (Tabla I). Se solicitaron voluntarios que no presentaran antecedentes clínicos que pudieran alterar el peso corporal o el metabolismo y, por tanto, los resultados. Después de un examen médico general, sólo fueron incluidos aquellos que habían mantenido su peso relativamente estable durante los últimos 3 meses y excluidos aquellos que hubiesen consumido fármacos o suplementos para la disminución del peso corporal, la inhibición del apetito o que aceleraran el metabolismo o consumido drogas que afectaran la temperatura corporal o la función cardiovascular. Después de explicados todos los procedimientos de la investigación, los participantes firmaron un consentimiento informado, como requisito para participar en el estudio. En él, a todos se les realizaron mediciones de TI, en condiciones ambientales controladas, para medir la temperatura de la piel, así como evaluaciones antropométricas y del GER. El protocolo experimental fue aprobado por la Comisión de Doctorado de la Universidad de León y siguió las recomendaciones descritas en la declaración de Helsinki para estudio con seres humanos.

Protocolo experimental. Los participantes asistieron a dos sesiones. Primera sesión; examen físico-médico y de la historia clínica de los sujetos. Se entregaron las indicaciones a seguir durante los días previos a las mediciones: a) continuar con sus actividades cotidianas, pero absteniéndose de realizar ejercicio intenso durante las 48 horas previas; b) no colocar en su piel cremas o ungüentos cosméticos; y, c) no consumir alimentos, líquidos y/o suplementos de tipo diuréticos, excitantes o fármacos. Se les solicitó, además, qué llegaran en ayuno nocturno y con 8 horas de sueño. Al final de esta sesión se realizó una familiarización con el equipo de medición de consumo de oxígeno, para evitar los efectos de ansiedad durante las mediciones. Segunda sesión; primero y siempre por las mañanas, se midió el GER y la TI en una sala y luego, en otra, se realizaron las mediciones antropométricas. Ambas salas se aclimataron entre los 23$25^{\circ} \mathrm{C}$ (Compher et al., 2006; Ammer \& Ring, 2012) y se utilizó calefacción por radiación, manteniendo la humedad relativa del aire entre el 60-70\%, apagándola durante cada recogida de datos de $\mathrm{TI}$.

Gasto energético en Reposo (GER). El GER fue medido a través de calorimetría indirecta de circuito abierto, usando un ergoespirómetro de respiración a respiración, modelo Metalyzer 3B-R2 (Cortex-Medical, Leipzig, Germany). Previo a la medición de cada sujeto se procedió a la calibración del equipo, para presión ambiental, volumen y gas. Durante la medición, los participantes permanecieron recostados en posición supino sobre una camilla, en condiciones de reposo, relajados y despiertos. Durante la evaluación, se colocó una máscara naso-bucal (Hans Rudolph, Kansas City, USA) a la que se conectó una turbina y una línea de muestra para detección de volúmenes de aire exhalado e intercambio de gases. Una vez alcanzado el estado estable del sujeto, se midieron los gases durante $20 \mathrm{~min}$. El nivel de estabilidad se identificó clínicamente a través de los siguientes criterios: cuando en un período de 5 minutos, el promedio por minuto de VO2 y VCO2 se modificó menos de un $10 \%$ y, cuando, el cociente respiratorio promedio se modificó menos de un $5 \%$ (Compher et al.). Cada 2 evaluaciones del GER, la sala era ventilada durante $1 \mathrm{~min}$.

Termografía por infrarrojo (TI). Después de 15 min de aclimatación en posición sentado, se procedió a recoger las imágenes termográficas para evaluar las zonas correspondientes a los pliegues cutáneos utilizados para la medición de la composición corporal (Fig. 1). Se utilizó una cámara térmica FLIR i7 (FLIR System, Oregon, USA), con una lente de ajuste automático, rango espectral entre 7,5 y $13 \mathrm{~mm}$ y 

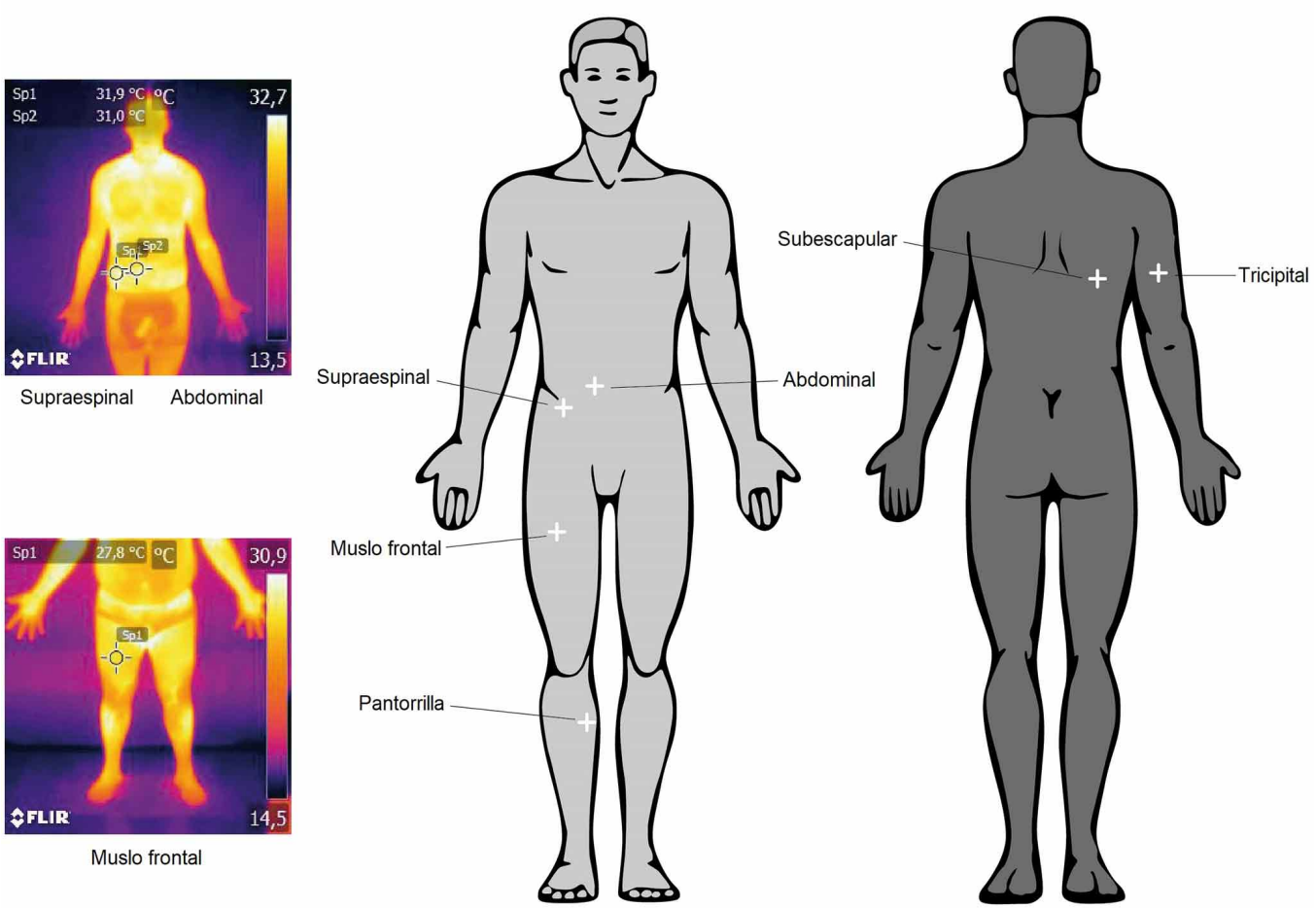

Fig. 1. Zonas corporales de medición de la termografía superficial por TI y pliegues cutáneos. Gris claro: vista anterior, Gris oscuro: vista posterior.

una sensibilidad térmica de $0,1^{\circ} \mathrm{C}$. Para el análisis de las imágenes termográficas se utilizó el software FLIR, tools+ (FLIR System, Oregon, USA). Durante el procedimiento los participantes se encontraban vestidos sólo con ropa interior y en posición anatómica. La cámara se ubicó de forma perpendicular a la zona evaluada y a una distancia entre los 95$100 \mathrm{~cm}$ de la superficie de la piel (Fernández-Cuevas et al.).

Mediciones antropométricas. Los datos antropométricos generales recogidos fueron el peso corporal, la estatura y la talla sentado, los 6 pliegues (tricipital, subescapular, supraespinal, abdominal, muslo medio y pantorrilla), 10 perímetros (cabeza, brazo relajado, brazo contraído, antebrazo, tórax, cintura, cadera, muslo máximo, muslo medio y pantorrilla) y 6 diámetros corporales (biacromial, tórax transverso, tórax anteroposterior, bi-iliocrestidio, humeral y femoral). Un antropometrista certificado (nivel II) aplicó el protocolo de medición estandarizado por la Sociedad Internacional para el Avance de la Cineantropometría (ISAK, International Society for the Advancement of Kinanthropometry) (Stewart et al., 2011). El peso corporal se obtuvo con una balanza electrónica, con sensibilidad de 20g, Jadever (JWI 3000®, Taiwan). La estatura y talla sentado se midieron con un estadiómetro Seca 213 (Seca, Alemania). El índice de masa corporal (IMC) fue obtenido dividiendo el peso corporal en kilogramos por la estatura en metros al cuadrado. Para medir los pliegues se utilizó un calibre Harpenden (Modelo
0120, Harpenden ${ }^{\circledR}$, England). Los perímetros se midieron con una cinta antropométrica metálica flexible Lufkin (W606PM, México). Para la medición de los diámetros se utilizaron dos antropómetros deslizantes de tamaño grande y pequeño, pertenecientes a un kit de medición antropométrico modelo Gaucho (Rosscraft $®$, USA). Para cuantificar los porcentajes de masa adiposa (MA\%), masa muscular (MM\%) y masa residual (MR\%) se utilizó el método de estimación de la composición corporal de Kerr (1988). Además, se obtuvieron indicadores antropométricos asociados al riesgo cardiometabólico, tales como el perímetro de cintura (PC), el índice cintura cadera (ICC) y el índice cintura estatura (ICE).

Análisis estadístico. Para el análisis de los datos y la confección de los gráficos se utilizó el software GraphPad Prism 7. Los resultados de antropometría y características generales son presentados como promedio y desviación estándar. Se utilizó la prueba de Shapiro-Wilk para observar la normalidad de distribución de los datos. Para las asociaciones que utilizaron las variables de índices de tejido muscular/ adiposo y muscular + residual/ adiposo, porcentaje del tejido muscular y sumatoria de pliegues cutáneos, se utilizó el coeficiente de correlación de Spearman, para las demás se utilizó el coeficiente de correlación de Pearson. Para todos los casos, se exigió un valor mínimo de $\mathrm{p}<0,05$ para ser considerado estadísticamente significativo. 

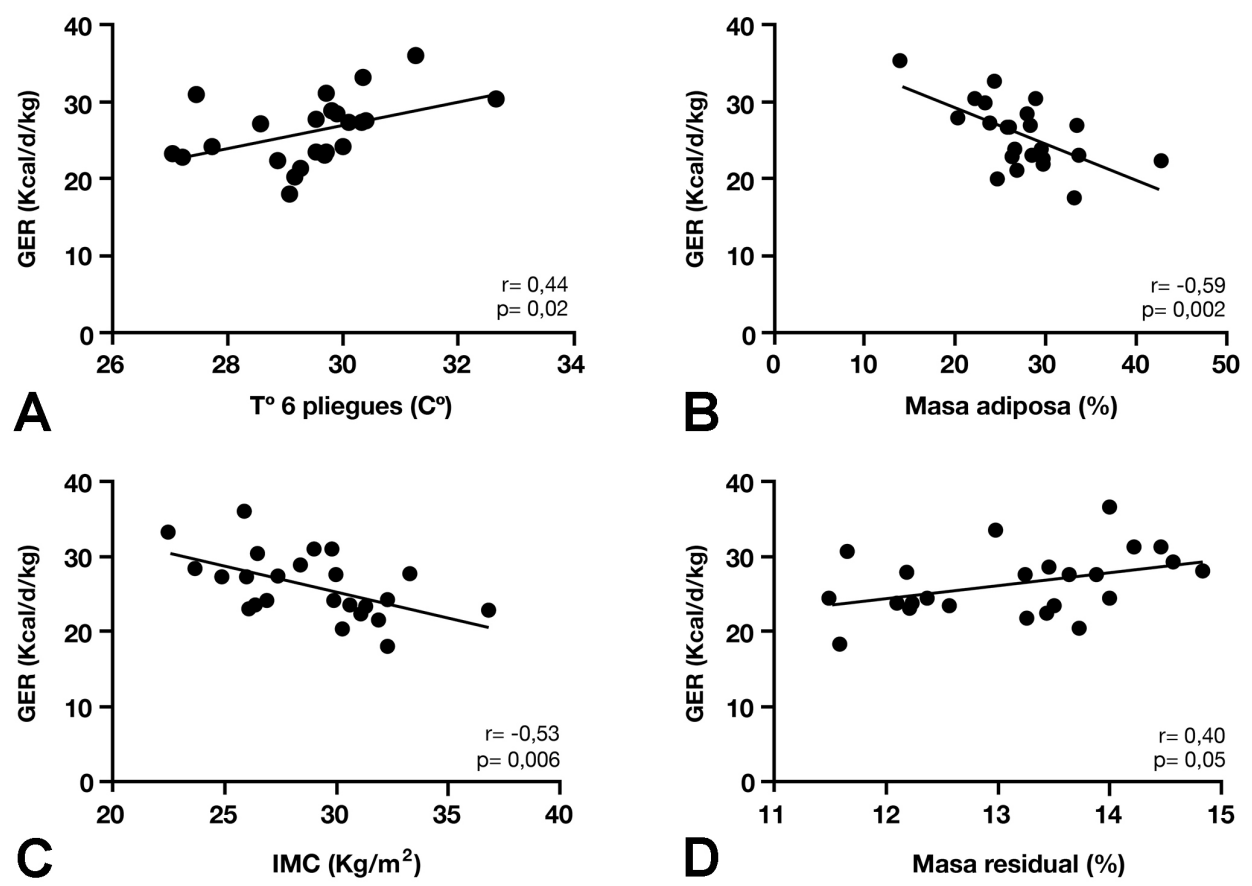

Fig. 2. Correlaciones entre el GER y el promedio de la temperatura superficial de los 6 pliegues (A), la masa adiposa (B), el índice de masa corporal (C) y la masa residual (D).

\section{RESULTADOS}

En la Tabla I se observan las características generales y antropométricas de los sujetos que han participado en el estudio.

En la Tabla II se observa que el pliegue subescapular, supra-espinal y pantorrilla tuvieron una correlación negativa $(\mathrm{p}<0,05)$ con la temperatura superficial del correspondiente pliegue, con $\mathrm{r}=-0,47(\mathrm{p}=0,018), \mathrm{r}=-0,50(\mathrm{p}=0,01)$ y $\mathrm{r}=-0,52(\mathrm{p}=0,001)$, respectivamente.

En la Tabla III se observa que la temperatura promedio de los 6-pliegues, y las individuales, toracoabdominales supra-espinal y abdominal, tuvieron correlaciones negati-
Tabla I. Características generales y antropométricas de la muestra. $\Sigma$-6p: Sumatoria de 6 pliegues cutáneos; IMC: Índice de masa corporal; PC: Perímetro de cintura; ICC: Índice cintura cadera; ICE: Índice cintura estatura; MA: Masa adiposa; MM: Masa muscular; MR: Masa residual; DE: Desviación estándar

\begin{tabular}{lc}
\hline & Promedio \pm DE \\
\hline Edad (años) & $33,9 \pm 8,7$ \\
Peso corporal $(\mathrm{kg})$ & $85,8 \pm 9,7$ \\
Estatura $(\mathrm{cm})$ & $172,5 \pm 6,1$ \\
$\Sigma-6 \mathrm{p}(\mathrm{cm})$ & $108,8 \pm 45,5$ \\
IMC $\left(\mathrm{kg} / \mathrm{m}^{2}\right)$ & $28,9 \pm 3,4$ \\
PC $(\mathrm{cm})$ & $92,8 \pm 8,3$ \\
ICC & $0,91 \pm 0,1$ \\
ICE & $0,54 \pm 0,06$ \\
MA $(\%)$ & $27,5 \pm 5,4$ \\
MM $(\%)$ & $44,6 \pm 4,1$ \\
MR $(\%)$ & $13,1 \pm 0,9$ \\
\hline
\end{tabular}

Tabla II. Correlaciones entre las mediciones antropométricas de los pliegues cutáneos y la temperatura superficial por termografía por infrarrojo (TI) de cada pliegue.

\begin{tabular}{|c|c|c|c|c|c|c|c|}
\hline \multirow{2}{*}{\multicolumn{2}{|c|}{ Temperatura por $\mathrm{TI}$}} & \multicolumn{6}{|c|}{ Medición antropometría de pliegues cutáneos (mm) } \\
\hline & & Tríceps & Subescapular & Supra-espinal & Abdominal & Muslo frontal & Pantorrilla \\
\hline \multirow{6}{*}{ TI } & Tríceps & $-0,22$ & -- & -- & -- & -- & -- \\
\hline & Subescapular & -- & $-0,47 *$ & -- & -- & -- & -- \\
\hline & Supra-espinal & -- & -- & $-0,50 *$ & -- & -- & -- \\
\hline & Abdominal & -- & -- & -- & $-0,27$ & -- & -- \\
\hline & Muslo frontal & -- & -- & -- & -- & $-0,34$ & -- \\
\hline & Pantorrilla & -- & -- & -- & -- & -- & $-0,52 * *$ \\
\hline
\end{tabular}

TI: termografía por infrarrojo. $(*) p<0,05 ;(* *) p<0,01$. 
vas con el IMC, PC, ICC, ICE, y la sumatoria de los 6 pliegues $(\mathrm{p}<0,05)$. La temperatura promedio de los 6 pliegues también mostró correlación negativa con la MA\% ( $\mathrm{p}=0,0269)$. La temperatura del pliegue supra-espinal tuvo una correlación negativa con la MA\% $(\mathrm{p}=0,0194)$ y positiva con la MM\% ( $\mathrm{p}=0,0461)$. Además, la temperatura de los pliegues tríceps, subescapular, supraespinal, abdominal y pantorrilla tuvieron correlaciones negativas con el PC, con $\mathrm{p}=0,0297, \mathrm{p}=0,0043, \mathrm{p}=0,005, \mathrm{p}=0,0010$ y $\mathrm{p}=0,0062$, respectivamente. Asimismo, las temperaturas de los pliegues supraespinal, abdominal y pantorrilla también mostraron correlaciones negativas con el ICE, con $\mathrm{p}=0,0037, \mathrm{p}=0,0031$ y $\mathrm{p}=0,0154$, respectivamente. Por último, la temperatura supraespinal, abdominal y pantorrilla también mostraron una correlación negativa con el IMC, con $\mathrm{p}=0,0149, \mathrm{p}=0,0138 \mathrm{y}$ $\mathrm{p}=0,0068$, respectivamente.

Respecto al GER, en la Figura 2 se observa que la MA\% y el IMC mostraron correlaciones negativas con el GER, con $r=-0,59(p=0,002)$ y $r=-0,53,(p=0,006)$, respectivamente, y positiva con el promedio de temperatura de los 6-pliegues con $r=0,44(p=0,02)$.

Tabla III. Correlaciones entre el promedio de la temperatura $\left(\mathrm{T}^{\circ}\right)$ por termografía por infrarrojo (TI) y parámetros antropométricos.

\begin{tabular}{|c|c|c|c|c|c|c|c|c|c|}
\hline \multirow[b]{2}{*}{$\mathrm{T}^{\circ}$ por $\mathrm{TI}\left({ }^{\circ} \mathrm{C}\right)$} & \multicolumn{9}{|c|}{ Parámetros antropométricos } \\
\hline & (MM/MA) & $\begin{array}{c}(\mathrm{MM}+\mathrm{MR}) / \\
\mathrm{MA}\end{array}$ & $\begin{array}{l}\text { MA } \\
(\%)\end{array}$ & $\begin{array}{l}\mathrm{MM} \\
(\%)\end{array}$ & $\begin{array}{l}\text { IMC } \\
\left(\mathrm{kg} / \mathrm{m}^{2}\right)\end{array}$ & $\begin{array}{l}\mathrm{PC} \\
(\mathrm{cm})\end{array}$ & ICC & ICE & $\begin{array}{l}\Sigma-6 \mathrm{p} \\
(\mathrm{mm})\end{array}$ \\
\hline Tríceps & 0,29 & 0,26 & $-0,29$ & 0,28 & $-0,35$ & $-0,44 *$ & $-0,27$ & $-0,34$ & $-0,31$ \\
\hline Subescapular & 0,26 & 0,22 & $-0,36$ & 0,26 & $-0,38$ & $-0,56^{*}$ & $-0,40$ & $-0,42 *$ & $-0,27$ \\
\hline Supra-es pinal & 0,38 & 0,32 & $-0,47 *$ & $0,41 * *$ & $-0,49 *$ & $-0,65^{*}$ & $-0,46^{* *}$ & $-0,57 * *$ & $-0,48 * *$ \\
\hline Abdominal & 0,28 & 0,23 & $-0,37$ & 0,33 & $-0,50 *$ & $-0,63 *$ & $-0,52 * *$ & $-0,58 * *$ & $-0,42 * *$ \\
\hline Muslo frontal & 0,20 & 0,20 & $-0,25$ & 0,22 & $-0,29$ & $-0,33$ & $-0,21$ & $-0,29$ & $-0,28$ \\
\hline Pantorrilla & 0,12 & 0,11 & $-0,32$ & 0,19 & $-0,54 *$ & $-0,54 *$ & $-0,31$ & $-0,49 *$ & $-0,20$ \\
\hline $\mathrm{T}^{\circ}-6 \mathrm{p}$ & 0,32 & 0,29 & $-0,45^{*}$ & 0,32 & $-0,54 * *$ & $-0,69 *$ & $-0,48^{*}$ & $-0,59 *$ & $-0,45 * *$ \\
\hline
\end{tabular}

$\mathrm{T}^{\circ}$ : Temperatura; TI: Termografía por infrarrojo; $\mathrm{T}^{\circ} 6 \mathrm{p}$ : Temperatura promedio de los 6 pliegues cutáneos; MM: Masa muscular; MA: Masa adiposa; MR: Masa residual; IMC: Índice de masa corporal; PC: Perímetro de cintura; ICC: Índice cintura cadera; ICE: Índice cintura estatura; -6p: Sumatoria de los 6 pliegues cutáneos $(*) \mathrm{p}<0.05,(* *) \mathrm{p}<0.01$.

\section{DISCUSIÓN}

Este estudio investigó la relación entre los parámetros de la antropometría, TI en las zonas de medición de los pliegues cutáneos y el GER. Nosotros observamos que la TI superficial promedio (brazo, tronco, muslo y pierna) es afectada por la acumulación de grasa corporal subcutánea total (sumatoria 6-pliegues), el MA\% y el estado ponderal (IMC), así como también por los indicadores de riesgo metabólico (PC, ICC, e ICE). Observamos que un mayor estado ponderal, dependiente de un aumento de la masa adiposa, se relaciona con un menor GER, mostrando una menor temperatura superficial en estos sujetos. Al igual que en Neves et al., nosotros no observamos correlación entre el pliegue del tríceps y la temperatura de la piel en la misma zona. En nuestro estudio sólo el pliegue subescapular, supra-espinal y pantorrilla, afectaron la TI de superficie. Esto podría demostrar el efecto que tiene la acumulación de grasa subcutánea sobre los patrones de liberación de calor superficial en el cuerpo de los sujetos. Es reconocido el efecto de la disminución en superficie, de la capacidad de disipación del calor corporal central a través del abdomen, en sujetos con mayor contenido de grasa corporal, observándose un aumento de la liberación de calor en las palmas de las manos (Savastano et al., 2009). Cabe recordar que la grasa corporal en el hombre tiende a acumularse en la zona abdominal (obesidad androide), provocando un mayor efecto de aislamiento con el exterior y causando un mayor descenso de la temperatura superficial en esta zona. En efecto, nosotros observamos una relación negativa con la MG\% y el estado ponderal, con la temperatura superficial individual, registrada sólo a nivel abdominal y supra-espinal. Así también, el tronco, es la principal zona de producción de calor corporal en reposo, debido al elevado metabolismo que tienen las vísceras toracoabdominales (Chudecka \& Lubkowska), de lo cual también el hígado, riñones y corazón contribuyen significativamente con el GER (Hwaung et al., 2019). En nuestro estudio, observamos una tendencia hacia una correlación positiva moderada entre la MR\% y GER $(\mathrm{p}=0,05)$ y que, en parte, evidencia este efecto. Ahora bien, a nivel abdominal también existe el efecto de la acumulación de la grasa visceral cuando nuestro estado ponderal incrementa, la que puede ser medida de forma directa a través de DXA o absorciometría de rayos X de energía dual (Shepherd et al., 2017). Desde el punto de vista 
antropométrico, el PC ha sido asociado con un incremento de la grasa visceral, y ha sido utilizado, para el control de la salud cardiovascular y metabólica en prevención primaria (Almeida et al., 2018). En nuestro estudio, el aumento del PC fue la variable antropométrica que obtuvo la mayor relación (alta) negativa con la temperatura superficial total y con las obtenidas individualmente, en las zonas abdominal y supra-espinal. Por ello, no sólo esta relación permite justificar un mayor efecto aislante de la grasa visceral en la capacidad de pérdida de calor hacia el exterior, sino que, además, la temperatura de la zona abdominal superficial podría ser a futuro, una alternativa de estudio para el riesgo cardiovascular en diferentes situaciones en el que se observa desorden metabólico (obesidad, cardiopatía, síndrome metabólico, entre otros).

Otro punto de interés en nuestro estudio, y que consideramos novedoso, fue la relación positiva observada entre la temperatura superficial promedio y el GER. Previamente, Carrasco et al. habían observado también una disminución en el GER en la medida que el estado ponderal era mayor, tal como ocurrió en nuestro estudio. Además, nosotros observamos que la MA\% tuvo una relación negativa moderada con el GER. Asimismo, la MM\% tuvo una relación positiva y la MR\% una tendencia positiva con el GER, similar a lo observado por el grupo de PriegoQuesada et al. (2019) y Heymsfield et al. (2012), respectivamente. Por lo tanto, aquellos sujetos con mayor cantidad de tejidos reconocidos como metabólicamente más activos (vísceras y músculo) y con menos de los metabólicamente poco activos (masa adiposa), tuvieron un mayor GER, el cual se relacionó con una mayor temperatura corporal superficial. Es por ello que, a medida que los tejidos de estos sujetos invertían su composición, el GER disminuyó, tal como ocurrió con la temperatura superficial promedio. De esta forma, sujetos aparentemente sanos desde un punto de vista metabólico y con parámetros antropométricos normales, con una mayor producción de calor derivado de la actividad metabólica de vísceras y músculos, muestran una menor resistencia a la disipación del calor hacia el exterior, incrementando la temperatura corporal promedio registrada en la piel en condiciones ambientales estables. En de interés que en nuestro estudio, se produjo el efecto contrario cuando los factores antropométricos de riesgo cardiovascular y metabólico se veían incrementados. En conclusión, los cambios en la composición corporal causan una modificación en los patrones de temperatura de la superficie de la piel medida por TI, sin afectar la relación entre el promedio de la temperatura superficial total con el GER, pudiendo este ser un factor predictor. Por ello, estimamos que puede crearse un modelo matemático predictor aumentando el volumen de muestra de estudio.
BARRAZA-GÓMEZ, F.; ALVEAR-ORDENES, I.; HECHTCHAU, G.; HENRÍQUEZ, M. \& TUESTA, M. Association between infrared thermography, anthropometry parameters and rest energy expenditure in men. Int. J. Morphol., 39(4):1176-1182, 2021.

SUMMARY: It is known that an increase in the subcutaneous body fat can alter the pattern of heat release through the skin. However, the relationship with other anthropometric variables and the influence of the Resting Energy Expenditure (REE) have scarcely been addressed. To determine the relationship of skin temperature with different anthropometric variables, body weight, and the REE of healthy adult males. The participants were a total of 24 healthy males of $33.9 \pm 8.7$ years-old, $85.8 \pm 9.7 \mathrm{~kg}$ of body mass, $172.5 \pm 6.1 \mathrm{~cm}$ of height, and $28.9 \pm 3.4 \mathrm{~kg} / \mathrm{m}^{2}$ of body mass index (BMI). Anthropometric, infrared thermography, and indirect calorimetry REE assessments were performed with gas analyzer. The six skinfolds average temperature, as well as the individual skinfolds (thoracoabdominal, supraspinal, and abdominal), had negative correlations with the BMI, waist circumference, waisthip ratio, waist-to-height ratio, and the sum of the six skinfolds, all with $\mathrm{p}<0.05$. In addition, the temperature of the supraspinal fold had a negative correlation with the percentage of fat mass (MA\%), $\mathrm{r}=0.47(\mathrm{p}=0.0194) . \mathrm{MA} \%$ and BMI had negative correlations with REE, with $\mathrm{r}=-0.59(\mathrm{p}=0.002)$ and $\mathrm{r}=-0.53(\mathrm{p}=0.006)$, respectively. The six-skinfolds average temperature presented a positive correlation with the REE ( $\mathrm{r}=0.44 ; \mathrm{p}=0.02)$. Body composition changes cause a modification in the local surface thermography patterns without affecting the relationship between the average total body surface temperature with the REE; therefore, this could act as a predictive factor.

KEY WORDS: Thermometry; Skinfold thickness; Metabolism; Body mass index.

\section{REFERENCIAS BIBLIOGRÁFICAS}

Almeida, E.; Pinho, C.; Leite, A.; Rodrigues, I.; Diniz, A. \& Arruda, I. Razón entre grasa visceral y subcutánea como predictor de alteraciones cardio-metabólicas. Rev. Chil. Nutr., 45(1): 28-36, 2018.

Ammer, K. \& Ring, F. J. Standard Procedures for Infrared Imaging in Medicine. In: Diakides, M.; Bronzino, J. D. \& Peterson, D. R. Medical Infrared Imaging. Principles and Practices. Boca Raton, CRC Press, 2012.

Bauer, J.; Hoq, M. N.; Mulcahy, J.; Tofail, S. A. M.; Gulshan, F.; Silien, C.; Podbielska, H. \& Akbar, M. M. Implementation of artificial intelligence and non-contact infrared thermography for prediction and personalized automatic identification of different stages of cellulite. EPMA J., 11:1729,2020

Carrasco, N. F.; Reyes, S. E.; Núñez, B. C.; Riedemann, S. K.; Rimler, S. O.; Sánchez, G. G. \& Sarrat, G. G. Gasto energético de reposo medido en obesos y no obesos: comparación con la estimación por fórmulas y ecuaciones propuestas para población chilena. Rev. Med. Chil., 130(1):51-60, 2002.

Chudecka, M. \& Lubkowska, A. Thermal maps of young women and men. Infrared Phys. Technol., 69:81-7, 2015.

Chudecka, M.; Zaborski, D.; Lubkowska, A.; Grzesiak, W.; Klimek, A. \& Modrzejewski, A. Temperature changes in selected areas of body surface induced by systemic cryostimulation. Aviat. Space Environ. Med., 85(12):1170-6, 2014 
Compher, C.; Frankenfield, D.; Keim, N. \& Roth-Yousey, L. Best practice methods to apply to measurement of resting metabolic rate in adults: A systematic review. J. Am. Diet. Assoc., 106(6):881-903, 2006.

Fernández-Cuevas, I.; Bouzas Marins, J. C.; Arnáiz Lastras, J.; Gómez Carmona, P. M.; Piñonosa Cano, S.; García-Concepción, M. Á. \& Sillero-Quintana, M. Classification of factors influencing the use of infrared thermography in humans: A review. Infrared Phys. Technol., 71:28-55, 2015.

Gutiérrez-Vargas, R.; Ugalde-Ramírez, J. A.; Rojas-Valverde, D.; SalasCabrera, J.; Rodríguez-Montero, A. \& Gutiérrez-Vargas, J. C. La termografía infrarroja como herramienta efectiva para detectar áreas músculares dañadas después de correr una maratón. Rev. Fac. Med., 65(4):601-7, 2017.

Hadzic, V.; Sirok, B.; Malnersic, A. \& Coh, M. Can infrared thermography be used to monitor fatigue during exercise? A case study. J. Sport Health Sci., 8(1):89-92, 2019.

Herman, C. \& Cetingul, M. P. Quantitative visualization and detection of skin cancer using dynamic thermal imaging. J. Vis. Exp., (51):2679, 2011.

Heymsfield, S. B.; Thomas, D.; Bosy-Westphal, A.; Shen, W.; Peterson, C. M. \& Müller, M. J. Evolving concepts on adjusting human resting energy expenditure measurements for body size. Obes. Rev., 13(11):1001-14, 2012.

Hidalgo Salvador, E.; Álvarez González, F. \& Salvador Luna, A. Aplicación de la termografía infrarroja en medicina legal. ¿Prueba válida para la objetivación de los síndromes dolorosos?. Disfunción temporomandibular. Cuad. Med. Forense, 20(2-3):77-84, 2014.

Hwaung, P.; Bosy-Westphal, A.; Muller, M. J.; Geisler, C.; Heo, M.; Thomas, D. M.; Kennedy, S. \& Heymsfield, S. B. Obesity tissue: composition, energy expenditure, and energy content in adult humans. Obesity (Silver Spring), 27(9):1472-81, 2019.

Jensen, M. M.; Poulsen, M. K.; Alldieck, T.; Larsen, R. G.; Gade, R.; Moeslund, T. B. \& Franch, J. Estimation of energy expenditure during treadmill exercise via thermal imaging. Med. Sci. Sports Exerc., 48(12):2571-9, 2016.

Kerr, D. A. An Anthropometric Method for Fractionation of Skin, Adipose, Bone, Muscle and Residual Tissue Masses, in Males and Females Age 6 to 77 years. Master of Science in Kinesiology. Burnaby, Simon Fraser University, 1988.

Law, J.; Morris, D. E.; Izzi-Engbeaya, C.; Salem, V.; Coello, C.; Robinson, L.; Jayasinghe, M.; Scott, R.; Gunn, R.; Rabiner, E.; et al. Thermal imaging is a noninvasive alternative to PET/CT for measurement of brown adipose tissue activity in humans. J. Nucl. Med., 59(3):516-22, 2018.

Neves, E. B.; Vilaça-Alves, J.; Nogueira, I. R. A. \& Reis, V. M. H. Influence of subcutaneous fat layer in skin temperature. Motricidade, 11:120-6, 2015.

Priego-Quesada, J. I.; Oficial-Casado, F.; Gandia-Soriano, A. \& Carpes, F. P. A preliminary investigation about the observation of regional skin temperatures following cumulative training loads in triathletes during training camp. J. Therm. Biol., 84:431-8, 2019.

Salamunes, A. C. C.; Stadnik, A. M. W. \& Neves, E. B. The effect of body fat percentage and body fat distribution on skin surface temperature with infrared thermography. J. Therm. Biol., 66:1-9, 2017.

Savastano, D. M.; Gorbach, A. M.; Eden, H. S.; Brady, S. M.; Reynolds, J. C. \& Yanovski, J. A. Adiposity and human regional body temperature. Am. J. Clin. Nutr., 90(5):1124-31, 2009.

Shepherd, J. A.; Ng, B. K.; Sommer, M. J. \& Heymsfield, S. B. Body composition by DXA. Bone, 104:101-5, 2017.

Stewart, A.; Marfell-Jones, M.; Olds, T. \& De Ridder, H. International Standards for Anthropometric Assessment. 3rd ed. Lower Hutt, International Society for Advancement of Kinanthropometry, 2011.

Weigert, M.; Nitzsche, N.; Kunert, F.; Lösch, C. \& Schulz, H. The influence of body composition on exercise-associated skin temperature changes after resistance training. J. Therm. Biol., 75:112-9, 2018.

\author{
Dirección para correspondencia: \\ Ildefonso Alvear-Ordenes. \\ FISAP, Instituto de Biomedicina (IBIOMED) \\ Campus de Vegazana, s/n 24071 \\ León \\ ESPAÑA
}

\section{E-mail: ialvor@unileon.es}

Recibido : 12-04-2021

Aceptado: 29-05-2021

Fernando Barraza-Gómez, https://orcid.org/0000-0002-3519-2108

Ildefonso Alvear-Ordenes, https://orcid.org/0000-0002-6870-4843

Gernot Hecht-Chau, https://orcid.org/0000-0002-8880-5190

Matías Henríquez, https://orcid.org/0000-0003-4392-1099

Marcelo Tuesta, https://orcid.org/0000-0002-5711-7520 